\title{
Computational Nutraceutics: Chemical Reactivity Properties of the Flavonoid Naringin by Means of Conceptual DFT
}

\author{
Jorge Ignacio Martínez-Araya, ${ }^{1}$ Guillermo Salgado-Morán, ${ }^{2}$ and Daniel Glossman-Mitnik ${ }^{3}$ \\ ${ }^{1}$ Dirección de Investigación y Desarrollo, Universidad Pedro de Valdivia, Avenida Tobalaba 1275, Santiago 7510275, Chile \\ ${ }^{2}$ Departamento de Ciencias, Facultad de Ciencias Exactas, Universidad Andrés Bello, Sede Concepción, Concepción 4070000, Chile \\ ${ }^{3}$ Laboratorio Virtual NANOCOSMOS, Centro de Investigación en Materiales Avanzados, SC, Miguel de Cervantes 120, \\ Complejo Industrial Chihuahua, 31109 Chihuahua, CHIH, Mexico
}

Correspondence should be addressed to Daniel Glossman-Mitnik; daniel.glossman@cimav.edu.mx

Received 27 January 2013; Revised 14 April 2013; Accepted 15 April 2013

Academic Editor: Juan S. Gómez-Jeria

Copyright (c) 2013 Jorge Ignacio Martínez-Araya et al. This is an open access article distributed under the Creative Commons Attribution License, which permits unrestricted use, distribution, and reproduction in any medium, provided the original work is properly cited.

\begin{abstract}
The M06 family of density functionals has been assessed for the calculation of the molecular structure and properties of the Naringin molecule. The chemical reactivity descriptors have been calculated through Conceptual DFT. The active sites for nucleophilic and electrophilic attacks have been chosen by relating them to the Fukui function indices and the dual descriptor $f^{(2)}(\mathbf{r})$. A comparison between the descriptors calculated through vertical energy values and those arising from the Koopmans' theorem approximation has been performed in order to check for the validity of the last procedure.
\end{abstract}

\section{Introduction}

The term nutraceutical is the result of a contraction between nutrition and pharmaceutical. In 1989, Dr. Stephen Defelice defined nutraceutical [1] as any substance that is a food or a part of a food and provides medical or health benefits, including the prevention and treatment of diseases [2]. In this work, we are coining the term "Computational Nutraceutics" for the practice of predicting the molecular structure, spectroscopy, and chemical reactivity of nutraceuticals by means of Computational Chemistry and Molecular Modeling.

The major active nutraceutical ingredients in plants are flavonoids. As is typical for phenolic compounds, they can act as potent antioxidants and metal chelators. They also have long been recognized to possess anti-inflammatory, antiallergic, hepatoprotective, antithrombotic, antiviral, and anticarcinogenic activities [3]. Naringin (C27H32O14; mol. wt. 581; IUPAC Name 7-[[2-O-(6-Deoxy- $\alpha$-L-mannopyranosyl)- $\beta$-D-glucopyranosyl]oxy]-2,3-dihydro-5-hydroxy-2(4-hydroxyphenyl)-4H-1-benzopyran-4-one) is a flavanone glycoside. It is a major flavonoid in grapefruit and gives the grapefruit juice its bitter taste. It is metabolized to the flavanone naringenin in humans. The aim of this work is to do a comparative study of the performance of the M06 family of density functionals for the description of the chemical reactivity of Naringin as a part of a continuing Computational Nutraceutics study of flavonoids.

The knowledge of reactivity on a molecule is an essential concept; it is of a crucial interest because it allows to understand interactions that are operating during a reaction mechanism. In particular electrostatic interactions have been successfully explained by the use of the molecular electrostatic potential $[4,5]$.

On the other hand, there is no a unique tool to quantify and rationalize covalent interactions; however, since 2005 a descriptor of local reactivity whose name is simply dual descriptor $[6,7]$ has allowed to rationalize reaction mechanisms in terms of overlapping nucleophilic regions with electrophilic regions in order to get a maximum stabilization thus leading to final products or intermediates; all those favorable nucleophilic-electrophilic interactions have been explained as a manifestation of the Principle of Maximum Hardness [8]; in addition, chemical reactions have been understood in terms of the Hard and Soft Acids and Bases 
Principle [9-14], the principle that has been used even with the aim of replacing the use of the molecular orbital theory to understand the whole chemistry [15]. In fact the present work is a good chance to test the capability of the most recent reactivity descriptors coming from the Conceptual DFT [1623]; therefore the framework of this conceptual theory will be presented in the next section.

\section{Theory and Computational Details}

At a local level, electronic density is the first local reactivity descriptor to be used when electrostatic interactions are predominant between molecules; within the framework of Conceptual DFT it is defined as follows:

$$
\rho(\mathbf{r})=\left[\frac{\delta E}{\delta v(\mathbf{r})}\right]_{N} .
$$

But when chemical reactions are governed by interactions mainly of covalent nature, in such a case a second-order local reactivity descriptor or LRD called Fukui function [18] is used instead of electronic density. Fukui function is defined in terms of the derivative of $\rho(\mathbf{r})$ with respect to $N$; through a Maxwell relation, the same descriptor is interpreted as the variation of $\mu$ with respect to $v(\mathbf{r})[18,24-26]$ as follows:

$$
f(\mathbf{r})=\left(\frac{\partial \rho(\mathbf{r})}{\partial N}\right)_{v(\mathbf{r})}=\left[\frac{\delta \mu}{\delta v(\mathbf{r})}\right]_{N} .
$$

The function $f(\mathbf{r})$ reflects the ability of a molecular site to accept or donate electrons. High values of $f(\mathbf{r})$ are related to a high reactivity at point $\mathbf{r}[18]$.

Since the number of electrons $N$ is a discrete variable [2729], right and left derivatives of $\rho(\mathbf{r})$ with respect to $N$ have emerged. Thus, two definitions of Fukui functions depending on total electronic densities are obtained as follows:

$$
\begin{aligned}
& f^{+}(\mathbf{r})=\left(\frac{\partial \rho(\mathbf{r})}{\partial N}\right)_{v(\mathbf{r})}^{+}=\rho_{N+1}(\mathbf{r})-\rho_{N}(\mathbf{r}), \\
& f^{-}(\mathbf{r})=\left(\frac{\partial \rho(\mathbf{r})}{\partial N}\right)_{v(\mathbf{r})}^{-}=\rho_{N}(\mathbf{r})-\rho_{N-1}(\mathbf{r}),
\end{aligned}
$$

where $\rho_{N+1}(\mathbf{r}), \rho_{N}(\mathbf{r})$ and $\rho_{N-1}(\mathbf{r})$ are the electronic densities at point $\mathbf{r}$ for the system with $N+1, N$, and $N-1$ electrons, respectively. The first one, $f^{+}(\mathbf{r})$, has been associated to reactivity for a nucleophilic attack, so that it measures the intramolecular reactivity at the site $\mathbf{r}$ toward a nucleophilic reagent. The second one, $f^{-}(\mathbf{r})$, has been associated to reactivity for an electrophilic attack, so that this function measures the intramolecular reactivity at the site $\mathbf{r}$ toward an electrophilic reagent [24].

The densities of frontier molecular orbitals (FMOs), $\rho_{L}(\mathbf{r})$ (LUMO density) and $\rho_{H}$ (r) (HOMO density), come to the picture since it has been shown $[24,30,31]$ that when the frozen orbital approximation (FOA) is used there is a direct relation between $f^{ \pm}(\mathbf{r})$ and the density of the appropriate
FMO thus avoiding calculations of the system with $N+1$ and $N-1$ electrons as follows:

$$
\begin{aligned}
& f^{+}(\mathbf{r}) \simeq \rho_{L}(\mathbf{r}), \\
& f^{-}(\mathbf{r}) \simeq \rho_{H}(\mathbf{r}) .
\end{aligned}
$$

On the other hand, the use of (4) instead of (3) allows one to diminish the computational effort without losing the qualitative picture of the local reactivity, but this approach should be always checked by comparison of these two couples of working equations because the first level of approximation based on total electronic densities will always be more accurate than the second level of approximation based on densities of FMOs.

Condensation to atoms is achieved through integration within the $k$ th-atomic domain $\Omega_{k}$ [32-37] as follows:

$$
f_{k}^{ \pm}=\int_{\Omega_{k}} f^{ \pm}(\mathbf{r}) d \mathbf{r}
$$

where $f_{k}^{ \pm}$is now an atomic index that is used to characterize the electrophilic/nucleophilic power of atom $k$.

Even much better, Morell et al. [7, 11, 15, 38-41] have proposed a local reactivity descriptor (LRD) which is called the dual descriptor (DD) $f^{(2)}(\mathbf{r}) \equiv \Delta f(\mathbf{r})$. In spite of having been discovered several years ago, a solid physical interpretation was not provided in such a moment [42]. They used the notation $\Delta f(\mathbf{r})$, but currently it has been replaced by the modern notation $f^{(2)}(\mathbf{r})$ in order to highlight that this is a Fukui function of second order. Its physical meaning is to reveal nucleophilic and electrophilic sites on a molecular system at the same time. Mathematically it is defined in terms of the derivative of the Fukui function, $f(\mathbf{r})$ [18], with respect to the number of electrons, $N$. Through a Maxwell relation, this LRD may be interpreted as the variation of $\eta$ (the molecular hardness which measures the resistance to charge transfer [43-45]) with respect to $v(\mathbf{r})$, the external potential. The definition of $f^{(2)}(\mathbf{r})$ is shown as indicated by Morell et al. $[7,11]$ as follows:

$$
f^{(2)}(\mathbf{r})=\left(\frac{\partial f(\mathbf{r})}{\partial N}\right)_{v(\mathbf{r})}=\left[\frac{\delta \eta}{\delta v(\mathbf{r})}\right]_{N} .
$$

As mentioned above, DD allows one to obtain simultaneously the preferably sites for nucleophilic attacks $\left(f^{(2)}(\mathbf{r})>0\right)$ and the preferably sites for electrophilic attacks $\left(f^{(2)}(\mathbf{r})<\right.$ 0 ) into the system at point $\mathbf{r}$. DD has demonstrated to be a robust tool to predict specific sites of nucleophilic and electrophilic attacks in a much more efficient way than the Fukui function by itself because dual descriptor is able to distinguish those sites of true nucleophilic and electrophilic behavior; in consequence some works have been published with the aim of remarking the powerfulness of $f^{(2)}(\mathbf{r})$ and all those LRDs depending on DD [7, 11, 15, 38-41].

The general working equation to obtain $\mathrm{DD}$ is given by the difference between nucleophilic and electrophilic Fukui function [15]. A well-known first level of approximation implies the use of finite difference method where the sum 
of electronic densities of the system with one more electron and one less electron is subtracted by the double of the total electronic density of the original system. Since this level of approximation implies a time-demanding computing, a second level of approximation has been used for some years where the densities of FMOs provide an easier-to-compute working equation as follows:

$$
f^{(2)}(\mathbf{r})=f^{+}(\mathbf{r})-f^{-}(\mathbf{r}) \simeq \rho_{L}(\mathbf{r})-\rho_{H}(\mathbf{r}),
$$

where densities of LUMO and HOMO are represented by $\rho_{L}(\mathbf{r})$ and $\rho_{H}(\mathbf{r})$, respectively.

Molecular symmetry can influence upon the local reactivity and on the other hand has been demonstrated that the Fukui function must conserve the symmetry [46, 47]. In addition, as the degeneration that may arise in frontier molecular orbitals is related with the molecular symmetry, within the framework of the second level of approximation, this phenomenon has been taken into account thus providing a mathematical expression to be applied on closed-shell molecular systems [48].

Hence, when an interaction between two species is well described through the use of this LRD, it is said that the reaction is controlled by frontier molecular orbitals (or frontier controlled) under the assumption that remaining molecular orbitals do not participate during the reaction [49].

The dual descriptor can also be condensed through an appropriate integration within the $k$ th-atomic domain $\Omega_{k}$ as follows:

$$
\int_{\Omega_{k}} f^{(2)}(\mathbf{r}) d \mathbf{r}=f_{k}^{(2)}
$$

When $f_{k}^{(2)}>0$ the process is driven by a nucleophilic attack on atom $k$, and then that atom acts as electrophilic species; conversely, when $f_{k}^{(2)}<0$ the process is driven by an electrophilic attack over atom $k$, therefore atom $k$ acts as a nucleophilic species.

\section{Settings and Computational Methods}

All computational studies were performed with the Gaussian 09 [50] series of programs with density functional methods as implemented in the computational package. The equilibrium geometries of the molecules were determined by means of the gradient technique. The force constants and vibrational frequencies were determined by computing analytical frequencies on the stationary points obtained after the optimization to check if there were true minima. The basis set used in this work was MIDIY, which is the same basis set as MIDI with a polarization function added to the hydrogen atoms. The MIDI basis is a small double-zeta basis (DZ) with polarization functions on N-F, Si-Cl, Br, and I [51-56]. The MIDI basis set and its derivatives have not attracted the attention that they deserve. However, based on our experience, these basis sets, combined with the proper density functional, are as good for the prediction of molecular properties as the usual DZ basis sets of the Pople's type.

For the calculation of the molecular structure and properties of the studied system, we have chosen the hybrid
meta-GGA density functionals M06, M06L, M06-2X, and $\mathrm{M} 06 \mathrm{HF}$ [57], which consistently provide satisfactory results for several structural and thermodynamic properties [57-59].

Within the conceptual framework of DFT [18, 43], the chemical potential $\mu$, which measures the escaping tendency of electron from equilibrium, is defined as

$$
\mu=\left(\frac{\partial E}{\partial N}\right)_{v(\vec{r})}=-\chi,
$$

where $\chi$ is the electronegativity.

The global hardness $\eta$ can be seen as the resistance to charge transfer as follows:

$$
\eta=\frac{1}{2}\left(\frac{\partial^{2} E}{\partial N^{2}}\right)_{v(\vec{r})} .
$$

Using a finite difference approximation and Koopmans' theorem [53-56], the previous expressions can be written as

$$
\begin{gathered}
\mu \approx-\frac{1}{2}(I+A) \approx \frac{1}{2}\left(\epsilon_{L}+\epsilon_{H}\right), \\
\eta \approx \frac{1}{2}(I-A) \approx \frac{1}{2}\left(\epsilon_{L}-\epsilon_{H}\right),
\end{gathered}
$$

where $\epsilon_{H}$ and $\epsilon_{L}$ are the energies of the highest occupied and the lowest unoccupied molecular orbitals, HOMO and LUMO, respectively. However, within the context of density functional theory, the previous inequalities are justified in light of the work of Perdew et al. [60], where they commented on the significance of the highest occupied Kohn-Sham eigenvalue and proved the ionization potential theorems for the exact Kohn-Sham density functional theory of a manyelectron system. In addition the use of the energies of frontier molecular orbitals as an approximation to obtain $I$ and $A$ is supported by the Janak's theorem [61]. In particular, the negative of Hartree-Fock and Kohn-Sham HOMO orbital has been found to define upper and lower limits, respectively, for the experimental values of the first ionization potential [62] thus validating the use of energies of Kohn-Sham frontier molecular orbital to calculate reactivity descriptors coming from Conceptual DFT [22, 37, 63].

The electrophilicity index $\omega$ represents the stabilization energy of the systems when it gets saturated by electrons coming from the surrounding

$$
\omega=\frac{\mu^{2}}{2 \eta} \approx \frac{(I+A)^{2}}{2(I-A)} \approx \frac{\left(\epsilon_{L}+\epsilon_{H}\right)^{2}}{2\left(\epsilon_{L}-\epsilon_{H}\right)} .
$$

The electron donating $\left(\omega^{-}\right)$and electron accepting $\left(\omega^{+}\right)$ powers have been defined as [64]

$$
\begin{aligned}
& \omega^{-}=\frac{(3 I+A)^{2}}{16(I-A)}, \\
& \omega^{+}=\frac{(I+3 A)^{2}}{16(I-A)} .
\end{aligned}
$$


It follows that a larger $\omega^{+}$value corresponds to a better capability of accepting charge, whereas a smaller value of $\omega^{-}$value of a system makes it a better electron donor. In order to compare $\omega^{+}$with $-\omega^{-}$, the following definition of net electrophilicity has been proposed [65]:

$$
\Delta \omega^{ \pm}=\omega^{+}-\left(-\omega^{-}\right)=\omega^{+}+\omega^{-}
$$

that is the electron accepting power relative to the electron donating power.

\section{Results and Discussion}

The molecular structure of Naringin was preoptimized by starting with the readily available PDB structure and finding the most stable conformer by means of the conformers module of Materials Studio through a random sampling with molecular mechanics techniques and a consideration of all the torsional angles. The structure of the resulting conformer was then optimized with the M06, M06L, M06-2X, and M06-HF density functionals in conjunction with the MIDIY basis set. The optimized molecular structure of the Naringin molecule (with the M06 density functional) is shown in Figure 1.

The validity of the Koopmans' theorem within the DFT approximation is controversial. However, it has been shown [62] that although the KS orbitals may differ in shape and energy from the HF orbitals, the combination of them produces Conceptual DFT reactivity descriptors that correlate quite well with the reactivity descriptors obtained through Hartree-Fock calculations. Thus, it is worth to calculate the electronegativity, global hardness, and global electrophilicity for the studied systems using both approximations in order to verify the quality of the procedures.

The HOMO and LUMO orbital energies (in eV), ionization potentials I and electron affinities A (in eV), and global electronegativity $\chi$, total hardness $\eta$, and global electrophilicity $\omega$ of the Naringin molecule calculated with the M06, M06L, M06-2X, and M06-HF density functionals and the MIDIY basis set are presented in Table 1. The upper part of the table shows the results derived assuming the validity of Koopmans' theorem, and the lower part shows the results derived from the calculated vertical $I$ and $A$. As can be seen from Table 1, the Koopman's theorem holds approximately for the density functionals which include some percentages of HF exchange, but it fails completely for the M06L density functional (without inclusion of HF exchange).

The condensed Fukui functions can also be employed to determine the reactivity of each atom in the molecule. The corresponding condensed functions are given by $f_{k}^{+}=q_{k}(N+$ 1) $-q_{k}(N)$ (for nucleophilic attack), $f_{k}^{-}=q_{k}(N)-q_{k}(N-1)$ (for electrophilic attack), and $f_{k}^{0}=\left[q_{k}(N+1)-q_{k}(N-1)\right] / 2$ (for radical attack), where $q_{k}$ is the gross charge of atom $k$ in the molecule.

It is possible to evaluate condensed Fukui functions from single-point calculations directly, without resorting to
TABLE 1: HOMO and LUMO orbital energies (in eV), ionization potentials $I$ and electron affinities $A$ (in eV), and global electronegativity $\chi$, total hardness $\eta$, and global electrophilicity $\omega$ of Naringin calculated with the M06, M06L, M06-2X, and M06-HF density functionals and the MIDIY basis set. The upper part of the table shows the results derived assuming the validity of Koopmans' theorem, and the lower part shows the results derived from the calculated vertical $I$ and $A$.

\begin{tabular}{lcccc}
\hline Property & M06 & M06L & M06-2X & M06-HF \\
\hline HOMO & -5.9078 & -4.6497 & -7.0972 & -9.0262 \\
LUMO & -0.6291 & -1.3507 & -1.6054 & -1.4177 \\
$\chi$ & 3.2685 & 3.0002 & 4.3513 & 5.2220 \\
$\eta$ & 2.6394 & 1.6495 & 2.7459 & 3.8043 \\
$\omega$ & 2.0238 & 5.8694 & 3.4477 & 3.5840 \\
\hline$I$ & 7.9980 & 8.2522 & 7.7756 & 8.3975 \\
$A$ & 0.7513 & 0.7309 & 0.8358 & 0.8151 \\
$\chi$ & 4.3747 & 4.4916 & 4.3057 & 4.6063 \\
$\eta$ & 3.6234 & 3.7607 & 3.4699 & 3.7912 \\
$\omega$ & 2.6409 & 2.6823 & 2.6714 & 2.7983 \\
\hline
\end{tabular}

additional calculations involving the systems with $N-1$ and $N+1$ electrons as follows:

$$
\begin{array}{cc}
f_{k}^{+}=\sum_{a \in k}\left[c_{a i}^{2}+c_{a i} \sum_{b \neq a} c_{b i} S_{a b}\right] \quad(\text { where } i=\mathrm{LUMO}), \\
f_{k}^{-}=\sum_{a \in k}\left[c_{a i}^{2}+c_{a i} \sum_{b \neq a} c_{b i} S_{a b}\right] & (\text { where } i=\mathrm{HOMO})
\end{array}
$$

with $c_{a i}$ being the LCAO coefficients and $S_{a b}$ being the overlap matrix. The condensed Fukui functions are normalized, thus $\sum_{k} f_{k}=1$ and $f_{k}^{0}=\left[f_{k}^{+}+f_{k}^{-}\right] / 2$.

The condensed Fukui functions have been calculated using the AOMix molecular analysis program [66, 67] starting from single-point energy calculations. We have presented, discussed, and successfully applied the described procedure in our previous studies on different molecular systems [6871].

The condensed dual descriptor has been defined as $f^{(2)}(\mathbf{r})_{k}=f_{k}^{+}-f_{k}^{-}[7,11]$. From the interpretation given to the Fukui function, one can note that the sign of the dual descriptor is very important to characterize the reactivity of a site within a molecule toward a nucleophilic or an electrophilic attack. That is, if $f^{(2)}(\mathbf{r})_{k}>0$, then the site is favored for a nucleophilic attack, whereas if $f^{(2)}(\mathbf{r})_{k}<0$, then the site may be favored for an electrophilic attack [7, 11, 72].

The electrophilic $f^{+}$and nucleophilic $f^{-}$condensed Fukui functions and $f^{(2)}(\mathbf{r})$ over the atoms of the Naringin molecule calculated with the M06, M06L, M06-2X, and M06HF density functionals and the MIDIY basis set are shown in Table 2 . The actual values have been multiplied by 100 for an easier comparison.

In Table 2, those atoms with a value for $f^{(2)}(\mathbf{r})$ close to zero are not shown. All four density functionals predict the same behavior. It can be concluded from the analysis of the results on Table 2 that $\mathrm{C} 8$ will be the preferred site for 


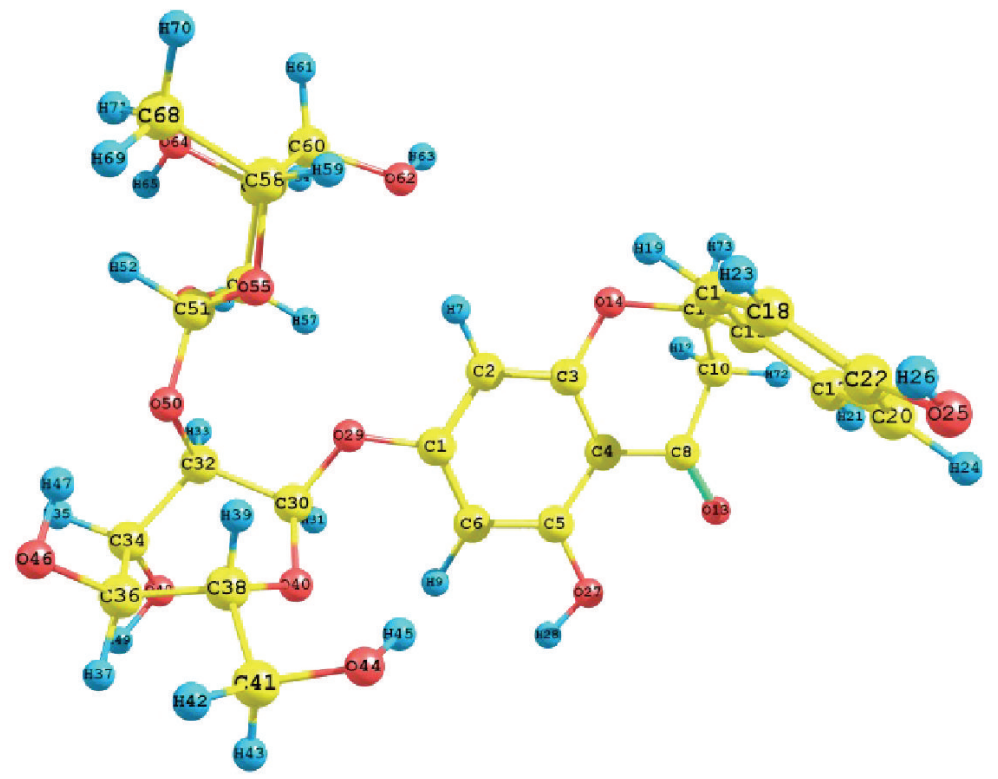

FIGURE 1: Optimized Molecular Structure of the Naringin Molecule.

TABLE 2: Electrophilic $f^{+}$and nucleophilic $f^{-}$condensed Fukui functions and $f^{(2)}(\mathbf{r})$ over the atoms of the Naringin molecule calculated with the M06, M06L, M06-2X, and M06-HF density functionals and the MIDIY basis set. The actual values have been multiplied by 100 for an easier comparison. $\mathrm{H}$ atoms are not shown.

\begin{tabular}{|c|c|c|c|c|c|c|c|c|c|c|c|c|}
\hline \multirow{2}{*}{ Atom } & \multicolumn{3}{|c|}{ M06 } & \multicolumn{3}{|c|}{ M06L } & \multicolumn{3}{|c|}{ M06-2X } & \multicolumn{3}{|c|}{ M06-HF } \\
\hline & $f^{+}$ & $f^{-}$ & $f^{(2)}(\mathbf{r})$ & $f^{+}$ & $f^{-}$ & $f^{(2)}(\mathbf{r})$ & $f^{+}$ & $f^{-}$ & $f^{(2)}(\mathbf{r})$ & $f^{+}$ & $f^{-}$ & $f^{(2)}(\mathbf{r})$ \\
\hline $1 \mathrm{C}$ & 16.48 & 0.65 & 15.83 & 15.04 & -0.01 & 15.05 & 17.17 & 3.06 & 14.11 & 18.49 & 2.81 & 15.68 \\
\hline $2 \mathrm{C}$ & 0.74 & 5.61 & -4.87 & 0.56 & 0.59 & -0.03 & 0.80 & 9.57 & -8.77 & 1.34 & 1.18 & 0.26 \\
\hline $3 C$ & 10.11 & 0.49 & 9.62 & 9.68 & 0.34 & 9.34 & 10.37 & 0.24 & 10.13 & 9.76 & 1.08 & 8.68 \\
\hline $4 \mathrm{C}$ & 6.17 & 6.03 & 0.14 & 8.58 & 4.66 & 3.92 & 7.71 & 7.45 & 0.26 & 10.56 & 6.45 & 4.13 \\
\hline $5 \mathrm{C}$ & 8.59 & 1.86 & 6.73 & 8.86 & 0.26 & 8.60 & 7.98 & 4.27 & 5.71 & 7.91 & 1.09 & 6.82 \\
\hline $6 \mathrm{C}$ & 1.54 & 1.54 & 0.00 & 0.94 & 0.51 & 0.43 & 2.04 & 0.46 & 1.58 & 2.76 & 1.00 & 1.76 \\
\hline $8 \mathrm{C}$ & 19.52 & 0.83 & 18.69 & 21.16 & 1.04 & 20.12 & 17.99 & 0.15 & 17.84 & 14.63 & 0.14 & 14.49 \\
\hline $10 \mathrm{C}$ & 0.25 & 6.13 & -5.88 & 0.23 & 10.45 & -10.22 & 0.27 & 0.52 & -0.25 & 0.28 & 0.31 & -0.03 \\
\hline $11 \mathrm{C}$ & 1.60 & 1.10 & 0.50 & 1.51 & 1.35 & 0.16 & 1.58 & 0.34 & 1.24 & 1.74 & 0.46 & 1.28 \\
\hline $13 \mathrm{O}$ & 17.18 & 32.39 & -15.21 & 18.96 & 69.06 & -50.10 & 2.07 & 16.45 & -14.38 & 1.04 & 14.47 & -13.43 \\
\hline $14 \mathrm{O}$ & 1.85 & 1.81 & 0.04 & 2.12 & 0.37 & 1.75 & 1.40 & 1.19 & 0.21 & 0.83 & 0.17 & 0.76 \\
\hline $15 \mathrm{C}$ & 0.41 & 8.88 & -8.47 & 0.24 & 0.73 & -0.49 & 0.50 & 17.02 & -16.52 & 0.69 & 24.01 & -23.32 \\
\hline $16 \mathrm{C}$ & 3.04 & 2.28 & 0.76 & 2.92 & 0.32 & 2.60 & 3.59 & 3.75 & -0.16 & 4.38 & 6.45 & -2.07 \\
\hline $17 \mathrm{C}$ & 0.26 & 1.29 & -1.03 & 0.31 & 0.19 & 0.12 & 0.37 & 2.57 & -2.20 & 0.53 & 3.23 & -2.70 \\
\hline $18 \mathrm{C}$ & 1.34 & 3.56 & -2.22 & 1.49 & 0.17 & 1.32 & 1.65 & 7.21 & -5.56 & 1.90 & 7.03 & -5.13 \\
\hline $20 \mathrm{C}$ & 2.09 & 5.11 & -3.02 & 2.09 & 0.48 & 1.61 & 2.66 & 8.28 & -5.62 & 3.34 & 11.58 & -8.24 \\
\hline $22 \mathrm{C}$ & 0.54 & 5.73 & -5.19 & 0.50 & 0.54 & -0.04 & 0.58 & 11.83 & -10.25 & 0.66 & 18.17 & -17.51 \\
\hline $25 \mathrm{O}$ & 0.14 & 8.16 & -8.02 & 0.15 & 0.72 & -0.57 & 0.11 & 11.92 & -11.81 & 0.08 & 10.56 & -10.48 \\
\hline $27 \mathrm{O}$ & 2.23 & 3.09 & -0.86 & 2.56 & 1.22 & 1.34 & 1.66 & 4.28 & -2.62 & 1.17 & 0.65 & 0.52 \\
\hline $29 \mathrm{O}$ & 2.62 & 0.74 & 1.88 & 2.94 & 0.05 & 2.89 & 2.22 & 2.31 & -0.09 & 1.58 & 1.14 & 0.34 \\
\hline
\end{tabular}

nucleophilic attack, while O13 will be the preferred site for electrophilic attack.

The electrodonating $\left(\omega^{-}\right)$and electroaccepting $\left(\omega^{+}\right)$powers and net electrophilicity $\Delta \omega^{ \pm}$of the Naringin molecule calculated with the M06, M06L, M06-2X, and M06-HF density functionals and the MIDIY basis set are presented in
Table 3. The upper part of the table shows the results derived assuming the validity of Koopmans' theorem, and the lower part shows the results derived from the calculated vertical $I$ and $A$.

The results from Table 3 clearly indicate that Naringin is an electrodonating molecule, with the same result predicted 
TABLE 3: Electrodonating $\left(\omega^{-}\right)$and electroaccepting $\left(\omega^{+}\right)$powers and net electrophilicity $\Delta \omega^{ \pm}$of Naringin calculated with the M06, M06L, M06-2X, and M06-HF density functionals and the MIDIY basis set. The upper part of the table shows the results derived assuming the validity of Koopmans' theorem, and the lower part shows the results derived from the calculated vertical $I$ and $A$.

\begin{tabular}{lcccc}
\hline Property & M06 & M06L & M06-2X & M06-HF \\
\hline$\omega^{-}$ & 3.9878 & 4.4348 & 5.9666 & 6.6704 \\
$\omega^{+}$ & 0.7194 & 1.4346 & 1.6152 & 1.4485 \\
$\Delta \omega^{ \pm}$ & 4.7072 & 5.8694 & 7.5818 & 8.1189 \\
\hline$\omega^{-}$ & 5.2810 & 5.3980 & 5.2580 & 5.5754 \\
$\omega^{+}$ & 0.9064 & 0.9065 & 0.9527 & 0.9691 \\
$\Delta \omega^{ \pm}$ & 6.1874 & 6.3045 & 6.2107 & 6.5445 \\
\hline
\end{tabular}

by all the four density functionals considered in this study. However, only the results obtained through the calculations with the M06, M06-2X, and M06-HF density functionals are in fairly agreement between those from vertical calculations of $I$ and $A$ and those coming from the assumption of the validity of the Koopmans' theorem in DFT.

\section{Conclusions}

From the whole results presented in this contribution it has been clearly demonstrated that the sites of interaction of the Naringin molecule can be predicted by using DFT-based reactivity descriptors such as the hardness, softness, and electrophilicity, as well as Fukui function calculations. These descriptors were used in the characterization and successfully description of the preferred reactive sites and provide a firm explanation for the reactivity of the Naringin molecule.

The M06 family of density functionals (M06, M06L, M06-2X, and M06-HF) used in the present work leads to the same qualitatively and quantitatively similar description of the chemistry and reactivity of the Naringin molecule, yielding reasonable results. However, for the case of the M06L functional, which does not include HF exchange, the agreement between the results obtained through energy calculations and those that assume the validity of of the Koopmans' theorem is definitively not true.

\section{Acknowledgments}

This work has been partially supported by CIMAV, SC, and Consejo Nacional de Ciencia y Tecnología (CONACYT, Mexico). Daniel Glossman Mitnik is a researcher of CONACYT and CIMAV.

\section{References}

[1] E. Kaira, "Nutraceutical-deffnition and introduction," AAPS Journal PharmScience, vol. 5, no. 3, pp. 27-28, 2003.

[2] A. Monge, "Functional foods.: reflexions of a scientist regarding a market in expansion," Revista de la Sociedad Química del Perú, vol. 74, no. 2, pp. 138-147, 2008.

[3] A. Tapas, D. Sakarkar, and R. Kakde, "Flavonoids as nutraceuticals: a review," Tropical Journal of Pharmaceutical Research, vol. 7, no. 3, pp. 1089-1099, 2008.
[4] P. Politzer and J. S. Murray, "The fundamental nature and role of the electrostatic potential in atoms and molecules," Theoretical Chemistry Accounts, vol. 108, no. 3, pp. 134-142, 2002.

[5] J. Murray and P. Politzer, "The electrostatic potential: an overview," WIREs Computational Molecular Science, vol. 1, pp. 153-163, 2011.

[6] C. Morell, A. Grand, and A. Toro-Labbé, "New dual descriptor for chemical reactivity," The Journal of Physical Chemistry A, vol. 109, no. 1, pp. 205-212, 2005.

[7] C. Morell, A. Grand, and A. Toro-Labbé, "Theoretical support for using the $\Delta \mathrm{f}(\mathbf{r})$ descriptor," Chemical Physics Letters, vol. 425, no. 4-6, pp. 342-346, 2006.

[8] R. G. Pearson, “The principle of maximum hardness," Accounts of Chemical Research, vol. 26, no. 5, pp. 250-255, 1993.

[9] R. G. Pearson, "Hard and soft acids and bases," Journal of the American Chemical Society, vol. 85, no. 22, pp. 3533-3539, 1963.

[10] R. G. Pearson, "Recent advances in the concept of hard and soft acids and bases," Journal of Chemical Education, vol. 64, no. 7, pp. 561-567, 1987.

[11] J. L. Gàzquez, "Hard and soft acids and bases principle," The Journal of Physical Chemistry A, vol. 101, no. 26, pp. 4657-4659, 1997.

[12] P. W. Ayers, R. G. Parr, and R. G. Pearson, "Elucidating the hard/soft acid/base principle: a perspective based on halfreactions," The Journal of Chemical Physics, vol. 124, no. 19, Article ID 194107, 8 pages, 2006.

[13] R. Pearson, Chemical Hardness, Wiley-VCH, New York, NY, USA, 1997.

[14] P. W. Ayers, "The physical basis of the hard/soft acid/base principle," Faraday Discussions, vol. 135, pp. 161-190, 2007.

[15] C. Cárdenas, N. Rabi, P. W. Ayers, C. Morell, P. Jaramillo, and P. Fuentealba, "Chemical reactivity descriptors for ambiphilic reagents: dual descriptor, local hypersoftness, and electrostatic potential," The Journal of Physical Chemistry A, vol. 113, no. 30, pp. 8660-8667, 2009.

[16] H. Chermette, "Density functional theory a powerful tool for theoretical studies in coordination chemistry," Coordination Chemistry Reviews, vol. 178-180, no. 1, pp. 699-721, 1998.

[17] H. Chermette, "Chemical reactivity indexes in density functional theory," Journal of Computational Chemistry, vol. 20, no. 1, pp. 129-154, 1999.

[18] P. Geerlings, F. de Proft, and W. Langenaeker, "Conceptual density functional theory," Chemical Reviews, vol. 103, no. 5, pp. 1793-1873, 2003.

[19] Y. E. Zevatskii and D. V. Samoilov, "Some modern methods for estimation of reactivity of organic compounds," Russian Journal of Organic Chemistry, vol. 43, no. 4, pp. 483-500, 2007.

[20] P. Johnson, L. Bartolotti, P. Ayers, T. Fievez, and P. Geerlings, "Charge density and chemical reactivity: a uniffed view from Conceptual DFT," in Modern Charge Density Analysis, C. Gatti and P. Macchi, Eds., pp. 715-764, Springer, New York, NY, USA, 2012.

[21] P. Geerlings, P. Ayers, A. Toro-Labbè, P. Chattaraj, and F. de Proft, "The woodward-homann rules reinterpreted by conceptual density functional theory," Accounts of Chemical Research, vol. 45, pp. 683-695, 2012.

[22] P. W. Ayers, J. S. M. Anderson, and L. J. Bartolotti, "Perturbative perspectives on the chemical reaction prediction problem," International Journal of Quantum Chemistry, vol. 101, no. 5, pp. 520-534, 2005. 
[23] J. L. Gázquez, "Perspectives on the density functional theory of chemical reactivity," Journal of the Mexican Chemical Society, vol. 52, no. 1, pp. 3-10, 2008.

[24] R. G. Parr and W. Yang, "Density functional approach to the frontier-electron theory of chemical reactivity," Journal of the American Chemical Society, vol. 106, no. 14, pp. 4049-4050, 1984.

[25] P. Ayers, W. Yang, and L. Bartolotti, "Fukui function," in Chemical Reactivity Theory: A Density Functional View, P. K. Chattaraj, Ed., chapter 18, pp. 255-267, CRC \& Taylor \& Francis, Boca Raton, Fla, USA, 2009.

[26] P. W. Ayers and M. Levy, "Perspective on 'Density functional approach to the frontier-electron theory of chemical reactivity," Theoretical Chemistry Accounts, vol. 103, no. 3-4, pp. 353-360, 2000.

[27] P. W. Ayers, "The dependence on and continuity of the energy and other molecular properties with respect to the number of electrons," Journal of Mathematical Chemistry, vol. 43, no. 1, pp. 285-303, 2008.

[28] J. P. Perdew, R. G. Parr, M. Levy, and J. L. Balduz Jr., "Densityfunctional theory for fractional particle number: derivative discontinuities of the energy," Physical Review Letters, vol. 49, no. 23, pp. 1691-1694, 1982.

[29] W. Yang, Y. Zhang, and P. W. Ayers, “Degenerate ground states and a fractional number of electrons in density and reduced density matrix functional theory," Physical Review Letters, vol. 84, no. 22, pp. 5172-5175, 2000.

[30] W. Yang, R. G. Parr, and R. Pucci, "Electron density, Kohn-Sham frontier orbitals, and Fukui functions," The Journal of Chemical Physics, vol. 81, no. 6, pp. 2862-2863, 1984.

[31] M. H. Cohen, M. V. Ganduglia-Pirovano, and J. Kudrnovský, "Electronic and nuclear chemical reactivity," The Journal of Chemical Physics, vol. 101, no. 10, pp. 8988-8997, 1994.

[32] P. Fuentealba, P. Pérez, and R. Contreras, "On the condensed Fukui function," The Journal of Chemical Physics, vol. 113, no. 7, pp. 2544-2551, 2000.

[33] F. A. Bulat, E. Chamorro, P. Fuentealba, and A. Toro-Labbé, "Condensation of frontier molecular orbital fukui functions," The Journal of Physical Chemistry A, vol. 108, no. 2, pp. 342-349, 2004.

[34] W. Yang and W. J. Mortier, "The use of global and local molecular parameters for the analysis of the gas-phase basicity of amines," Journal of the American Chemical Society, vol. 108, no. 19 , pp. 5708-5711, 1986.

[35] P. W. Ayers, R. C. Morrison, and R. K. Roy, "Variational principles for describing chemical reactions: condensed reactivity indices," The Journal of Chemical Physics, vol. 116, no. 20, pp. 8731-8744, 2002.

[36] P. Bultinck, S. Fias, C. Alsenoy, P. Ayers, and R. CarbóDorca, "Critical thoughts on computing atom condensed Fukui functions," The Journal of Chemical Physics, vol. 127, Article ID 034102, 11 pages, 2007.

[37] F. Zielinski, V. Tognetti, and L. Joubert, "Condensed descriptors for reactivity: a methodological study," Chemical Physics Letters, vol. 527, pp. 67-72, 2012.

[38] A. Toro-Labbé, Ed., Theoretical Aspects of Chemical Reactivity, vol. 19, Elsevier, New York, NY, USA, 2007.

[39] P. W. Ayers, C. Morell, F. de Proft, and P. Geerlings, "Understanding the Woodward-Hoffmann rules by using changes in electron density," Chemistry, vol. 13, no. 29, pp. 8240-8247, 2007.
[40] C. Morell, P. W. Ayers, A. Grand, S. Gutiérrez-Oliva, and A. Toro-Labbé, "Rationalization of Diels-Alder reactions through the use of the dual reactivity descriptor $\Delta \mathrm{f}(\mathbf{r})$," Physical Chemistry Chemical Physics, vol. 10, no. 48, pp. 7239-7246, 2008.

[41] C. Morell, A. Hocquet, A. Grand, and B. Jamart-Grégoire, "A conceptual DFT study of hydrazino peptides: assessment of the nucleophilicity of the nitrogen atoms by means of the dual descriptor $\Delta \mathrm{f}(\mathbf{r})$," Journal of Molecular Structure, vol. 849, no. 13, pp. 46-51, 2008.

[42] P. Fuentealba and R. G. Parr, "Higher-order derivatives in density-functional theory, especially the hardness derivative $\partial \eta / \partial \mathrm{N}, "$ The Journal of Chemical Physics, vol. 94, no. 8, pp. 5559$5564,1991$.

[43] R. G. Parr and W. Yang, Density Functional Theory of Atoms and Molecules, Oxford University Press, New York, NY, USA, 1989.

[44] F. de Proft, P. W. Ayers, S. Fias, and P. Geerlings, "WoodwardHoffmann rules in density functional theory: initial hardness response," The Journal of Chemical Physics, vol. 125, no. 21, Article ID 214101, 9 pages, 2006.

[45] F. de Proft, P. K. Chattaraj, P. W. Ayers et al., "Initial hardness response and hardness profiles in the study of WoodwardHoffmann rules for electrocyclizations," Journal of Chemical Theory and Computation, vol. 4, no. 4, pp. 595-602, 2008.

[46] R. Flores-Moreno, "Symmetry conservation in fukui functions," Journal of Chemical Theory and Computation, vol. 6, no. 1, pp. 48-54, 2010.

[47] C. Cárdenas, P. W. Ayers, and A. Cedillo, "Reactivity indicators for degenerate states in the density-functional theoretic chemical reactivity theory," The Journal of Chemical Physics, vol. 134, no. 17, Article ID 174103, 13 pages, 2011.

[48] J. Martínez, "Local reactivity descriptors from degenerate frontier molecular orbitals," Chemical Physics Letters, vol. 478, no. 4-6, pp. 310-322, 2009.

[49] G. Klopman, "Chemical reactivity and the concept of chargeand frontier-controlled reactions," Journal of the American Chemical Society, vol. 90, no. 2, pp. 223-234, 1968.

[50] M. J. Frisch, G. W. Trucks, and H. B. Schlegel, Gaussian 09 Revision A.1, Gaussian, Wallingford, Conn, USA, 2009.

[51] S. Huzinaga, J. Andzelm, M. Klobulowski, E. Radzio-Audselm, Y. Sakai, and H. Tatewaki, Gaussian Basis Sets for Molecular Calculations, Elsevier, New York, NY, USA, 1984.

[52] R. E. Easton, D. J. Giesen, A. Welch, C. J. Cramer, and D. G. Truhlar, "The MIDI! basis set for quantum mechanical calculations of molecular geometries and partial charges," Theoretical Chemistry Accounts, vol. 93, no. 5, pp. 281-301, 1996.

[53] E. Lewars, Computational Chemistry - Introduction to the Theory and Applications of Molecular and Quantum Mechanics, Kluwer Academic, New York, NY, USA, 2003.

[54] D. C. Young, Computational Chemistry -A Practical Guide for Applying Techniques to Real-World Problems, John Wiley \& Sons, New York, NY, USA, 2001.

[55] F. Jensen, Introduction to Computational Chemistry, John Wiley \& Sons, New York, NY, USA, 2nd edition, 2007.

[56] C. J. Cramer, Essentials of Computational Chemistry - Theories and Models, John Wiley \& Sons, New York, NY, USA, 2nd edition, 2004.

[57] Y. Zhao and D. G. Truhlar, "Density functionals with broad applicability in chemistry," Accounts of Chemical Research, vol. 41, no. 2, pp. 157-167, 2008.

[58] Y. Zhao and D. G. Truhlar, "The M06 suite of density functionals for main group thermochemistry, thermochemical kinetics, 
noncovalent interactions, excited states, and transition elements: two new functionals and systematic testing of four M06-class functionals and 12 other functionals," Theoretical Chemistry Accounts, vol. 120, no. 1-3, pp. 215-241, 2008.

[59] Y. Zhao and D. G. Truhlar, "Applications and validations of the Minnesota density functionals," Chemical Physics Letters, vol. 502, no. 1-3, pp. 1-13, 2011.

[60] J. Perdew, K. Burke, and M. Ersernhof, "Errata: generalized gradient approximation made simple," Physical Review Letters, vol. 78, p. 1396, 1997.

[61] J. F. Janak, "Proof that $\partial E \partial n_{i}=\varepsilon$ in density-functional theory," Physical Review B, vol. 18, no. 12, pp. 7165-7168, 1978.

[62] J. Zevallos and A. Toro-Labbé, "A theoretical analysis of the Hohn-Sham and Hartree-Fock orbitals and their use in the determination of electronic properties," Journal of the Chilean Chemical Society, vol. 48, no. 4, pp. 39-47, 2003.

[63] A. Savin, C. J. Umrigar, and X. Gonze, "Relationship of KohnSham eigenvalues to excitation energies," Chemical Physics Letters, vol. 288, no. 2-4, pp. 391-395, 1998.

[64] J. L. Gázquez, A. Cedillo, and A. Vela, "Electrodonating and electroaccepting powers," The Journal of Physical Chemistry A, vol. 111, no. 10, pp. 1966-1970, 2007.

[65] P. K. Chattaraj, A. Chakraborty, and S. Giri, "Net electrophilicity," The Journal of Physical Chemistry A, vol. 113, no. 37, pp. 10068-10074, 2009.

[66] S. Gorelsky, AOMix Program for Molecular Orbital AnalysisVersion 6.5, Ottawa, Canada, University of Ottawa, 2011, http://www.sg-chem.net/.

[67] S. I. Gorelsky and A. B. P. Lever, "Electronic structure and spectra of ruthenium diimine complexes by density functional theory and INDO/S. Comparison of the two methods," Journal of Organometallic Chemistry, vol. 635, no. 1-2, pp. 187-196, 2001.

[68] T. Ruiz-Anchondo and D. Glossman-Mitnik, "Computational characterization of the $\beta, \beta$-carotene molecule," Journal of Molecular Structure, vol. 913, no. 1-3, pp. 215-220, 2009.

[69] D. Glossman-Mitnik, "Computational study of 3,4-diphenyl1,2,5-thiadiazole 1-oxide for organic photovoltaics," International Journal of Photoenergy, vol. 2009, Article ID 806714, 8 pages, 2009.

[70] D. Glossman-Mitnik, "Computational molecular characterization of Coumarin-102," Journal of Molecular Structure, vol. 911, no. 1-3, pp. 105-108, 2009.

[71] T. Ruiz-Anchondo, N. Flores-Holguín, and D. GlossmanMitnik, "Natural carotenoids as nanomaterial precursors for molecular photovoltaics: a computational DFT study," Molecules, vol. 15, no. 7, pp. 4490-4510, 2010.

[72] J. L. Gàzquez, "Chemical reactivity concepts in density functional theory," in Chemical Reactivity Theory: A Density Functional View, P. K. Chattaraj, Ed., chapter 2, pp. 7-21, CRC \& Taylor \& Francis, Boca Raton, Fla, USA, 2009. 

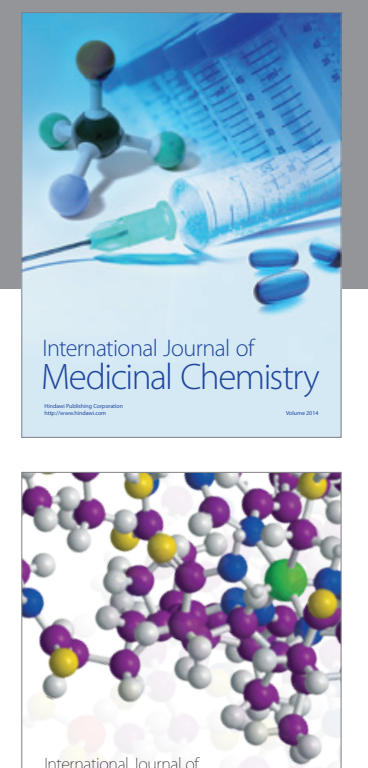

\section{Carbohydrate} Chemistry

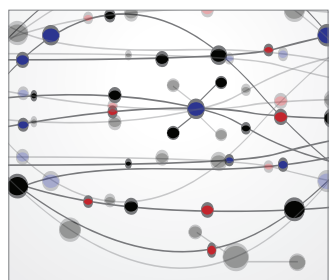

The Scientific World Journal
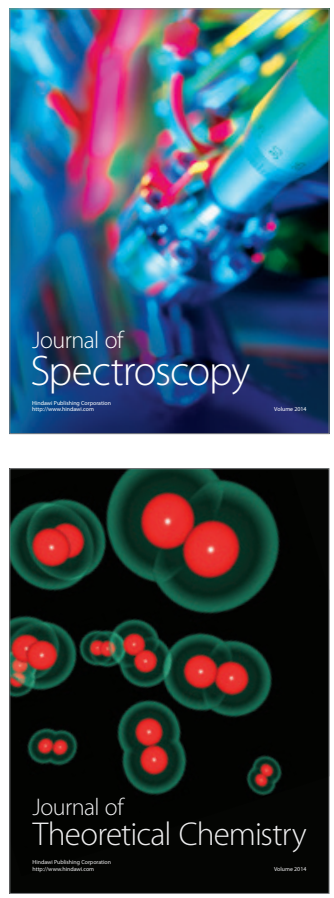
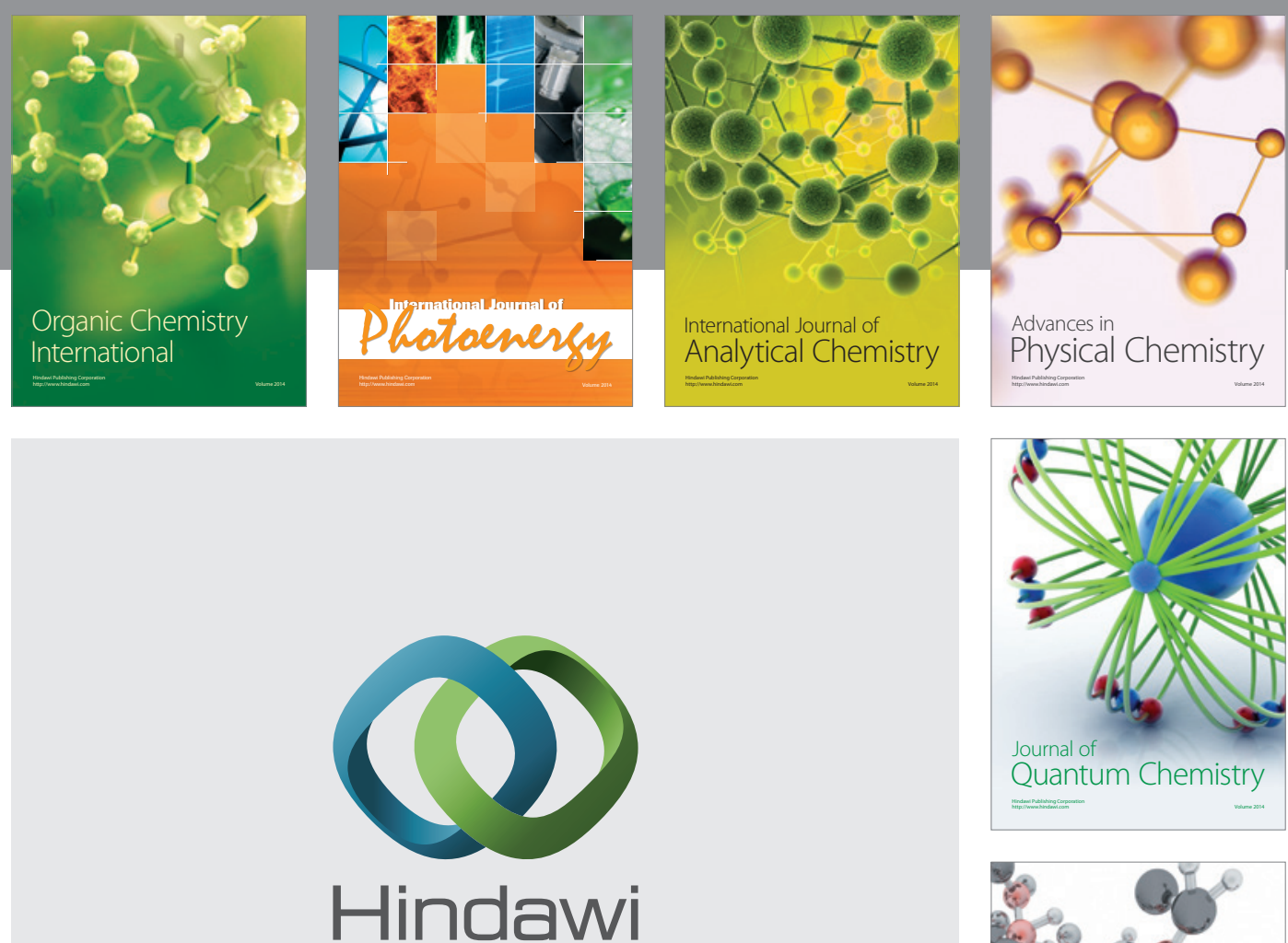

Submit your manuscripts at

http://www.hindawi.com

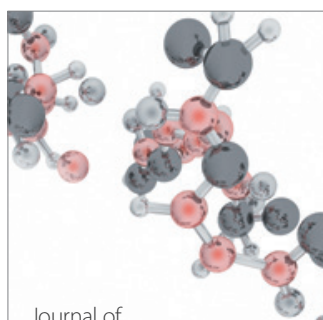

Analytical Methods

in Chemistry

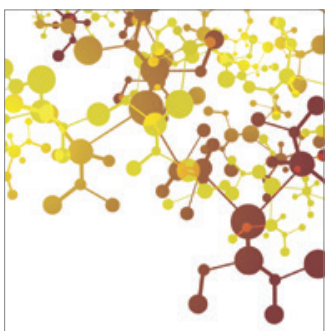

Journal of

Applied Chemistry

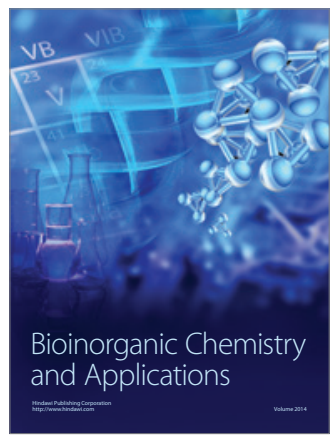

Inorganic Chemistry
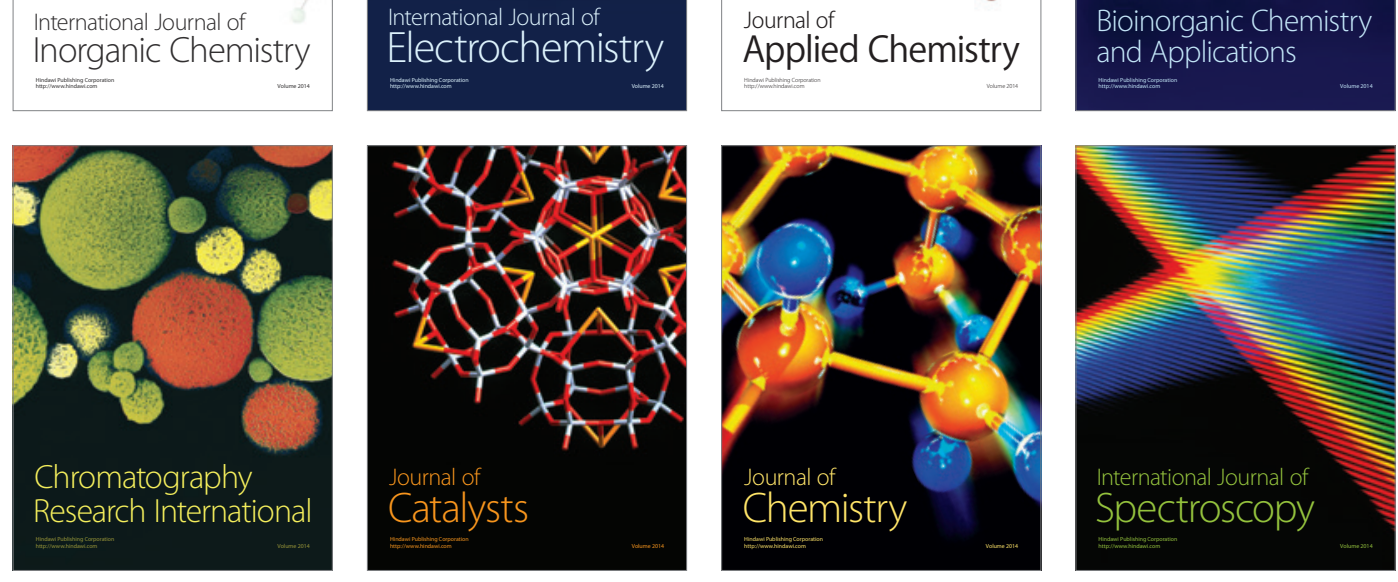